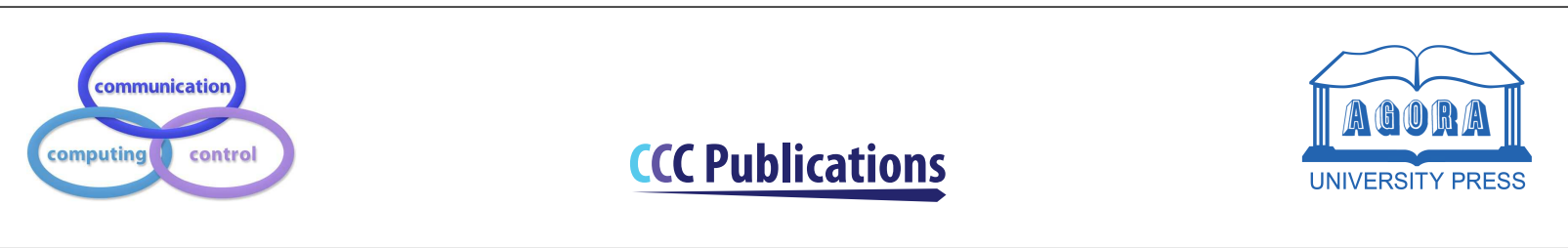

\title{
The Pseudo-Pascal Triangle of Maximum Deng Entropy
}

\author{
X. Gao, Y. Deng
}

\author{
Xiaozhuan Gao ${ }^{1}$ \\ Institute of Fundamental and Frontier Science \\ University of Electronic Science and Technology of China, Chengdu, 611731 \\ Yong Deng ${ }^{12 *}$ \\ 1. Institute of Fundamental and Frontier Science \\ University of Electronic Science and Technology of China, Chengdu, 611731 \\ 2. Medical Center, Vanderbilt University, Nashville, TN. USA, 37205 \\ *Corresponding author: dengentropy@uestc.edu.cn, prof.deng@hotmail.com
}

\begin{abstract}
Pascal triangle (known as Yang Hui Triangle in Chinese) is an important model in mathematics while the entropy has been heavily studied in physics or as uncertainty measure in information science. How to construct the the connection between Pascal triangle and uncertainty measure is an interesting topic. One of the most used entropy, Tasllis entropy, has been modelled with Pascal triangle. But the relationship of the other entropy functions with Pascal triangle is still an open issue. Dempster-Shafer evidence theory takes the advantage to deal with uncertainty than probability theory since the probability distribution is generalized as basic probability assignment, which is more efficient to model and handle uncertain information. Given a basic probability assignment, its corresponding uncertainty measure can be determined by Deng entropy, which is the generalization of Shannon entropy. In this paper, a Pseudo-Pascal triangle based the maximum Deng entropy is constructed. Similar to the Pascal triangle modelling of Tasllis entropy, this work provides the a possible way of Deng entropy in physics and information theory.

Keywords: Deng entropy, Maximum Deng Entropy, Pascal triangle, Dempster-Shafer evidence theory, basic probability assignment.
\end{abstract}

\section{Introduction}

Pascal triangle (known as the Yang Hui Triangle in China) is a triangular arrangement of the binomial coefficients and that it contains many remarkable numerical relations [19,64]. Pascal-Triangle has also become a suitable model system for the exploration of statistical mechanical structures [53, 64]. Pascal Triangle has attracted many people attention and been used in many fields $[45,46]$, such as network [28], cellular automata[79] and so on[5, 6, 43].

However, how to set connection between Pascal triangle and uncertainty is an interesting issue. In uncertainty, entropy plays an important role [8, 82] and has been used in many fields [9, 22, 58, 75]. There are various entropies, such as Shannon entropy, Renyi entropy [51], Kolmogorov entropy and so on. Hence, setting the connection between Pascal triangle and entropy is an issue with considering. 
Tsallis proposed the Tsallis entropy based on the probability theory as generalization of BoltzmannGibbs statistics [61] and analyzed the connection between Tsallis entropy and Pascal-triangle [62]. As the extension of probability theory, Dempster-Shafer evidence theory (D-S evidence theory) has attracted many people attention. About uncertainty in D-S evidence theory, there are also various measurement. Deng proposed Deng entropy to measure the uncertainty of D-S evidence theory $[10,57]$ ) [14], which is compatible with Shannon entropy. Deng entropy has attracted many people attention since proposed $[3,15,25,26,27,54,66]$, which has been used in many fields, such as information fusion [35, 47], quantum information [20], uncertainty measurement [41] and so on [63, 73]. Recently, Kang and Deng proposed the maximum Deng entropy, which BPA satisfies some conditions with the changes of frame of discernment [24]. Based on the maximum Deng entropy, the paper proposed the pseudo-Pascal triangle to further explore Deng entropy.

The paper is organized as follows. The preliminaries Dempster-Shafer evidence theory and Deng entropy are briefly introduced in Section 2. Section 3 introduced the maximum Deng entropy and discussed the some properties of BPA, besides, analysed the Semi-Pascal-Triangle of BPA. Finally, this paper is concluded in Section 4.

\section{Preliminaries}

In this section, the preliminaries of D-S theory $[10,57]$ and Deng entropy [14] will be briefly introduced.

\subsection{Dempster-Shafer evidence theory}

D-S evidence theory offers a useful fusion tool for uncertain information [18, 77]. D-S evidence theory needs weaker conditions than the Bayesian theory of probability, D-S evidence theory assigns the probability into the power set of events[16]. D-S evidence theory has been used to many applications, such as data fusion $[1,49,60]$, conflict management [33, 55], pattern recognition [44, 52, 56], evidential reasoning $[17,80,81]$ and so on $[2,21,34,74]$. Some preliminaries in D-S theory are introduced as follows. For additional details about D-S theory, refer to $[10,57]$.

Definition 1. ( Frame of discernment)

Let $\Theta$ be the set of mutually exclusive and collectively exhaustive events $A_{i}$, namely

$$
\Theta=\left\{A_{1}, A_{2}, \cdots, A_{n}\right\}
$$

The power set of $\Theta$ composed of $2^{N}$ elements of is indicated by $2^{\Theta}$, namely:

$$
2^{\Theta}=\left\{\phi,\left\{A_{1}\right\},\left\{A_{2}\right\}, \cdots,\left\{A_{1}, A_{2}\right\}, \cdots, \Theta\right\}
$$

Definition 2. ( Mass Function)

For a frame of discernment $\Theta=\left\{A_{1}, A_{2}, \cdots, A_{n}\right\}$, the mass function $\mathrm{m}$ is defined as a mapping of $m$ from 0 to 1, namely:

$$
m: 2^{\Theta} \rightarrow[0,1]
$$

which satifies

$$
\begin{gathered}
m(\phi)=0 \\
\sum_{A \subseteq \Theta} m(A)=1
\end{gathered}
$$

In D-S theory, a mass function is also called a basic probability assignment (BPA) or a piece of evidence or belief structure. The $m(A)$ measures the belief exactly assigned to A and represents how strongly the piece of evidence supports A. If $m(A)>0, \mathrm{~A}$ is called a focal element, and the union of all focal elements is called the core of a mass function. There are some studies about BPA, such as association [48], negation [40, 76], divergence [75] and information quality [7, 29, 30]. 


\subsection{Deng entropy}

Uncertainty modelling is still an open issue [32, 38, 39, 50, 59, 65]. Many models are presented to model the complexity such as network models [31, 68, 69, 70, 71], Bayesian [23, 42, 78] and D numbers $[12,13,36,37]$. Among many math tools and models, entropy function plays an important role in real applications [11, 72]. Deng proposed an Deng Entropy, which is an generalization of Shannon entropy [14].

Definition 3. (Deng entropy)

Given a BPA, Deng entropy can be defined as:

$$
H_{D}=-\sum_{A \subseteq \Theta} m(A) \log _{2} \frac{m(A))}{2^{|A|}-1}
$$

Through a simple transformation, Deng Entropy can be rewrite as follows:

$$
H_{D}=\sum_{A \subseteq \Theta} m(A) \log _{2}\left(2^{|A|}-1\right)-\sum_{A \subseteq \Theta} m(A) \log _{2} m(A)
$$

where $m$ is a BPA defined on the frame of discernment $\Theta$, and $A$ is the focal element of $\mathrm{m},|A|$ is the cardinality of $A$. Besides, the term $\sum m(A) \times \log _{2}\left(2^{|A|}-1\right)$ could be interpreted as a measure of total nonspecificity in the mass function $m$, and the term $-m(A) \times \log _{2} m(A)$ is the measure of discord of the mass function among various focal elements.

Definition 4. (The Maximum Deng entropy)

Given a BPA, the maximum Deng entropy is as follows:

$$
H_{M-D}=-\sum m(A) \times \log _{2} \frac{m(A)}{2^{|A|}-1}
$$

if and only if

$$
m(A)=\frac{2^{|A|}-1}{\sum 2^{|A|}-1}
$$

The more details about maximum Deng entropy refer to [24].

\section{The Pseudo-Pascal Triangle of Maximum Deng Entropy}

\subsection{The Example of Maximum Deng Entropy}

The specific BPA of having maximum Deng entropy was showed as follows.

Example 1. Given the frame of discernment $\Theta=\{A\}$, there is only one phenomenon, as follows:

$$
\begin{gathered}
m(A)=1 \\
H_{D}=0
\end{gathered}
$$

From the above, it can be easily found that only having one element means no uncertainty, that is to say, the event is certain.

Example 2. Given the frame of discernment $\Theta=\{A, B\}$, the BPA and maximum Deng entropy are shown below:

$$
\begin{aligned}
m(A)=\frac{1}{5}, m(B) & =\frac{1}{5}, m(A, B)=\frac{3}{5} \\
H_{M-D} & =2.3219
\end{aligned}
$$

From the above, the BPA of having maximum Deng entropy has the phenomenon that $m(A)$ and $m(B)$ has the same distribution. 
Example 3. Given the frame of discernment $\Theta=\{A, B, C\}$, the BPA and maximum Deng entropy are as follows:

$$
\begin{gathered}
m(A)=\frac{1}{19}, m(B)=\frac{1}{19}, m(C)=\frac{1}{19} \\
m(A, B)=\frac{3}{19}, m(B, C)=\frac{3}{19}, m(A, C)=\frac{3}{19}, m(A, B, C)=\frac{7}{19} \\
H_{M-D}=4.2474
\end{gathered}
$$

It can known that the mass function having the same cardinality has same distribution. Besides, from the above Example 1, Example 2 and Example 3, it can be seen that the BPA follow a certain

\begin{tabular}{|c|c|c|c|c|}
\hline Frame of Discrement & $|X|=1$ & $|X|=2$ & $|X|=3$ & $|X|=4$ \\
\hline$\Theta=\{A\}$ & 1 & & & \\
\hline$\Theta=\{A, B\}$ & $(1 / 5,1 / 5)$ & $3 / 5$ & & \\
\hline$\Theta=\{A, B, C\}$ & $(1 / 19,1 / 19,1 / 19)$ & $(3 / 19,3 / 19,3 / 19)$ & $7 / 19$ & \\
\hline$\Theta=\{A, B, C, D\}$ & $\begin{array}{c}(1 / 65,1 / 65,(3 / 65,3 / 65,3 / 65, \\
1 / 65,1 / 65) 3 / 65,3 / 65,3 / 65)\end{array}$ & $\begin{array}{l}(7 / 65,7 / 65, \\
7 / 65,7 / 65)\end{array}$ & & \\
\hline
\end{tabular}
rules with changes of frame of discernment. More details, it can be discussed in the next.

\subsection{Distribution of maximum Deng entropy}

Table 1: The BPA of having Maximum Deng entropy

Table 1 shows BPA of having maximum Deng entropy in various frame of discernment. In Table $1,|X|$ represents the cardinality of focal element, $\Theta$ represents the frame of discernment, the other information represents the BPA. Such as, for $\Theta=\{A, B\}$, in the second column, $\left(\frac{1}{5}, \frac{1}{5}\right)$ means the $m(A)=\frac{1}{5}$ and $m(B)=\frac{1}{5},\left(\frac{3}{5}\right)$ means the $m(A, B)=\frac{3}{5}$. From the Table 1, it can be known focal element having the same cardinality has same BPA in the case of maximum Deng entropy. Besides, the BPA can be rewrite the Fig.1. In Fig .1, the first column, $N$ corresponds to the number of frame of discernments. Besides, the second column corresponds to the BPA with having maximum Deng entropy. For example, in $(2,3 / 5), 2$ represents that the cardinality of focal element, $3 / 5$ represents the BPA.

$$
\begin{aligned}
& \mathrm{N}=1 \\
& \mathrm{~N}=2 \\
& \mathrm{~N}=3 \\
& \mathrm{~N}=4
\end{aligned}
$$

Figure 1: The BPA of having Maximum Deng entropy

\subsection{Pseudo-Pascal Triangle of maximum Deng entropy}

Based the above discussion, it can be seen that the BPA has some regularity. Fig, 2 shows the connection between Pseudo-Pascal triangle and the maximum Deng entropy. In Fig. 2, $N$ represents the number of frame of discernment. $|m|$ represents the cardinality of BPA, the others represents the number of having the $2^{|m|}-1$. For better understanding, it can be considered that a system can be divided with some subsystems. That is to say, a system is evenly distributed, every subsystem occupies a certain proportion. When the frame of Discernment is $N$, the system can be divided into $\sum_{m=1}^{m=N}\left(2^{|m|}-1\right) \times C_{N}^{m}$ parts, the focal elements whose cardinality is $|m|$ has $2^{|m|}-1$ parts.

From the Fig. 2, when the $N=2$, the system can be divided 5 parts. In this system, the number having $2^{|1|}-1=1$ parts is 2 , the number having $2^{|2|}-1=3$ parts is 1 . Through the above analyse, it can be known that the distribution of the maximum Deng entropy is similarly with Pascal triangle, 


\begin{tabular}{|c|c|c|c|c|c|c|c|c|c|c|c|c|}
\hline $2|\mathrm{~m}|-1$ & $2^{|1|-1}$ & $2^{|2|-1}$ & $2^{|3|-1}$ & $2^{|4|-1}$ & $2^{|5|-1}$ & $2^{|6|}-1$ & $2^{|7|-1}$ & $2^{|8|-1}$ & $2^{|9|-1}$ & $2^{|10|-1}$ & $2^{|11|-1}$ & $2^{|12|-1}$ \\
\hline $\mathrm{N}=1$ & 1 & & & & & & & & & & & \\
\hline $\mathrm{N}=2$ & 2 & 1 & & & & & & & & & & \\
\hline $\mathrm{N}=3$ & 3 & 3 & 1 & & & & & & & & & \\
\hline $\mathrm{N}=4$ & 4 & 6 & 4 & 1 & & & & & & & & \\
\hline $\mathrm{N}=5$ & 5 & 10 & 10 & 5 & 1 & & & & & & & \\
\hline $\mathrm{N}=6$ & 6 & 15 & 20 & 15 & 6 & 1 & & & & & & \\
\hline $\mathrm{N}=7$ & 7 & 21 & 35 & 35 & 21 & 7 & 1 & & & & & \\
\hline $\mathrm{N}=8$ & 8 & 28 & 56 & 70 & 56 & 28 & 8 & 1 & & & & \\
\hline $\mathrm{N}=9$ & 9 & 36 & 84 & 126 & 126 & 84 & 36 & 9 & 1 & & & \\
\hline $\mathrm{N}=10$ & 10 & 45 & 120 & 210 & 252 & 210 & 120 & 45 & 10 & 1 & & \\
\hline $\mathrm{N}=11$ & 11 & 55 & 165 & 330 & 462 & 462 & 330 & 165 & 55 & 11 & 1 & \\
\hline $\mathrm{N}=12$ & 12 & 66 & 220 & 495 & 792 & 924 & 792 & 495 & 220 & 66 & 12 & 1 \\
\hline
\end{tabular}

Figure 2: The Pseudo-Pascal Triangle

called Pseudo-Pascal triangle. Hence, the paper proposed the connection between Pseudo-Pascal triangle and the maximum Deng entropy.

\section{Conclusions}

Pascal triangle is an important tool in mathematics to deal with some issue. Uncertainty plays an essential role in modern lief. Hence, how to generate the connection between Pascal triangle and uncertainty is an interesting issue. Deng entropy can provide a method to measure the uncertainty in D-S evidence theory, which is compatible with Shannon entropy. The paper discussed the BPA of the maximum Deng entropy, which changes with $N$ according to evolutionary rules. By discussion, it can be found that the BPA of the maximum Deng entropy can construct Pseudo-Pascal triangle. The paper analysis the BPA of maximum Deng entropy, which is useful for uncertainty measurement.

The paper have set the relationship between Pseudo-Pascal triangle and maximum Deng entropy. However, the relationship between maximum Deng entropy and Pascal triangle still needs further exploration.

\section{Acknowledgment}

The work is partially supported by National Natural Science Foundation of China (Grant Nos. 61973332 ).

\section{References}

[1] Abellán, J.; Mantas, C.J.; Bossé, E.(2019). Basic Properties for Total Uncertainty Measures in the Theory of Evidence, Information Quality in Information Fusion and Decision Making, 99-108, 2019 .

[2] Abellán, J.; Mantas, C.J.; Castellano, J. G. (2017). A Random Forest approach using imprecise probabilities, Knowledge-Based Systems, 134, 72-84, 2017.

[3] Abellán, J. (2017). Analyzing properties of Deng entropy in the theory of evidence, Chaos Solitons 8 Fractals, 95, 195-199, 2017.

[4] Ahmia, M.; Belbachir, H.. (2012). Preserving log-convexity for generalized Pascal triangles, the electronic journal of combinatorics, 19(2), 16, 2012. 
[5] Becher, V.; Carton, O. (2019). Normal numbers and nested perfect necklaces, Journal of Complexity, 54, 101403, 2019.

[6] Blyth, M.G.; Pozrikidis, C. (2006). A lobatto interpolation grid over the triangle, IMA journal of applied mathematics, 71(1), 153-169, 2006.

[7] Cao, X.; Deng, Y. (2019). A lobatto interpolation grid over the triangle, IEEE ACCESS, 7(1), 95547-95554, 2019.

[8] Cao, Z; Ding, W.; Wang, Y.-K.; Hussain F., Al-Jumaily, A. Lin, C.-T. (2019). Effects of Repetitive SSVEPs on EEG Complexity using Multiscale Inherent Fuzzy Entropy, Neurocomputing, DOI: 10.1016/j.neucom.2018.08.091, 2019.

[9] Cao, Z.; Lin, C.-T. (2018). Inherent fuzzy entropy for the improvement of EEG complexity evaluation, IEEE Transactions on Fuzzy Systems, 26(2), 1032-1035, 2018.

[10] Dempster, A.P. (1967). Upper and Lower Probabilities Induced by a Multivalued Mapping, Annals of Mathematical Statistics, 38(2), 325-339, 1967.

[11] Deng, W.; Deng, Y. (2018). Entropic methodology for entanglement measures, Physica A: Statistical Mechanics and its Applications, 512, 693-697, 2018.

[12] Deng, X.; Jiang, W. (2019). Evaluating green supply chain management practices under fuzzy environment: a novel method based on D number theory, International Journal of Fuzzy Systems, 21, 1389-1402, 2019.

[13] Deng, X.; Jiang, W. (2019). A total uncertainty measure for D numbers based on belief intervals, International Journal of Intelligent Systems, 34(12), 3302-3316, 2019.

[14] Deng, Y. (2016). Deng Entropy, Chaos, Solitons \& Fractals, 91, 549-553, 2016.

[15] Dragan, I.-M.; Isaic-Maniu, A. (2019). An Innovative Model of Reliability-The Pseudo-Entropic Model, Entropy, 21(9), 846, 2019.

[16] Elmore, P. A.; Petry F.E. Yager, R.R. (2017). Dempster-Shafer Approach to Temporal Uncertainty, IEEE Transactions on Emerging Topics in Computational Intelligence, 1(5), 316-325, 2017.

[17] Fang, R.; Liao, H.; Yang, J.-B., Xu, D.-L. (2019). Generalised probabilistic linguistic evidential reasoning approach for multi-criteria decision-making under uncertainty, Journal of the Operational Research Society, DOI:10.1080/01605682.2019.1654415, 2019.

[18] Gao, S.; Deng, Y. (2019). An evidential evaluation of nuclear safeguards, International Journal of Distributed Sensor Networks, 15(12), DOI:10.1177/1550147719894550, 2019.

[19] Hacène, B.; Nèmeth, L.; Szalay, L.. (2016). Hyperbolic pascal triangles, Applied Mathematics and Computation, 273, 453-464, 2016.

[20] Huang, Z.; Yang, L.; Jiang, W. (2019). Uncertainty measurement with belief entropy on the interference effect in the quantum-like Bayesian Networks, Applied Mathematics and Computation, $347,417-428,2019$.

[21] Hurley, J.; Johnson, C.; Dunham, J.; Simmons, J. (2019). Nonlinear Algorithms for Combining Conflicting Identification Information in Multisensor Fusion, 2019 IEEE Aerospace Conference, $1-7,2019$.

[22] Jafferis, D. L.; Lewkowycz, A.; Maldacena, J.; Suh, S. J. (2016). Relative entropy equals bulk relative entropy, Journal of High Energy Physics, 2016(6), 4, 2016. 
[23] Jiang, W.; Cao, Y.; Deng, X. (2019). A Novel Z-network Model Based on Bayesian Network and Z-number, IEEE Transactions on Fuzzy Systems, DOI:10.1109/TFUZZ.2019.2918999, 2019.

[24] Kang, B.; Deng, Y. (2019). The maximum Deng entropy, IEEE ACCESS, 7(1), 120758-120765, 2019.

[25] Karci, A. (2016). Fractional order entropy: New perspectives, Optik, 127(20), 9172-9177, 2016.

[26] Khan, N.; Anwar, S. (2019). Time-Domain Data Fusion Using Weighted Evidence and DempsterShafer Combination Rule: Application in Object Classification, Sensors, 19(23), 5187, 2019.

[27] Kuzemsky, A. L. (2018). Temporal evolution, directionality of time and irreversibility, Rivista Del Nuovo Cimento, 41(10), 513-574, 2018.

[28] Lee, S.; Jin, M.; Koo, B.; Sin, C.; Kim, S. (2016). Pascal's triangle-based range-free localization for anisotropic wireless networks, Wireless Networks, 22(7), 2221-2238, 2016.

[29] Li, D.; Deng, Y. (2019). A new correlation coefficient based on generalized information quality, IEEE ACCESS, 7(1), 175411-175419, 2019.

[30] Li, D.; Deng, Y.; Gao, X. (2019). A generalized expression for information quality of basic probability assignment, IEEE ACCESS, 7(1), 174734-174739, 2019.

[31] Li, H.; He, Y.; Nie, X. (2018). Structural reliability calculation method based on the dual neural network and direct integration method, Neural Computing and Applications, 29(7), 425-433, 2018.

[32] Li, H.; Yuan, R.; Fu, J. (2019). A reliability modeling for multi-component systems considering random shocks and multistate degradation, IEEE ACCESS, 7(1), 168805-168814, 2019.

[33] Li, M.; Deng, Y. (2019). Evidential Decision Tree Based on Belief Entropy, Entropy, 21(9), 897, 2019.

[34] Li, Y.; Deng, Y. (2019). Intuitionistic evidence sets, IEEE ACCESS, 7(1), 106417-106426, 2019.

[35] Liu, F.; Gao, X.; Zhao, J.; Deng, Y. (2019). Generalized belief entropy and its application in identifying conflict evidence, IEEE ACCESS, 7(1), 126625-126633, 2019.

[36] Liu, P.; Zhang, X. (2019). A Multicriteria Decision-Making Approach with Linguistic D Numbers Based on the Choquet Integral, Cognitive Computation, DOI: 10.1007/s12559-019-09641-3, 2019.

[37] Liu, P.; Zhang, X.; Wang, Z. (2019). An Extended VIKOR Method for Multiple Attribute Decision Making with Linguistic D Numbers Based on Fuzzy Entropy, International Journal of Information Technology \& Decision Making, DOI: 10.1142/S0219622019500433, 2019.

[38] Liu, W.; Wang, T.; Zang, T.; Huang, Z.; Wang, J.; Huang, T.; Wei, X.; Li, C. (2020). A fault diagnosis method for power transmission networks based on spiking neural $\mathrm{P}$ systems with self-updating rules considering biological apoptosis mechanism, Complexity, DOI: $10.1155 / 2020 / 2462647,2020$.

[39] Liu, Y.; Jiang, W. (2019). A new distance measure of interval-valued intuitionistic fuzzy sets and its application in decision making, Complexity, 23, DOI:10.1007/s00500-019-04332-5, 2019.

[40] Liu, Z.; Deng, Y. (2019). A matrix method of basic belief assignment's negation in DempsterShafer theory, IEEE Transactions on Fuzzy Systems, 27, DOI:10.1109/TFUZZ.2019.2930027, 2019 .

[41] Mamb, M. D.; N'Takpe, T.; Anoh, N. G.; Oumtanaga, S. (2018). A New Uncertainty Measure in Belief Entropy Framework, International Journal of Advanced Computer Science and Applications, 9(11), 600-606, 2018. 
[42] Mi, J.; Li, Y. F.; Beer, M.; Broggi, M.; Cheng, Y. (2020). Importance measure of probabilistic common cause failures under system hybrid uncertainty based on Bayesian network, Eksploatacja i Niezawodnosc-Maintenance and Reliability, 13(22), 112-120, 2020.

[43] Millard, P.; Massou, S.; Portais, J.-C.; Letise, F. (2014). Isotopic studies of metabolic systems by mass spectrometry: using Pascal's triangle to produce biological standards with fully controlled labeling patterns, Analytical chemistry, 86(20), 10288-10295, 2014.

[44] Mo, H.; Deng, Y. (2019). Identifying node importance based on evidence theory in complex networks, Physica A: Statistical Mechanics \& Its Applications, DOI:10.1016/j.physa.2019.121538, 2019 .

[45] Moussa, A.; Hacène, B. (2012). Preserving log-convexity for generalized Pascal triangles, The electronic journal of combinatorics, 19(2), 16, 2012.

[46] Nemeth, L.; Szalay, L. (2018). Power sums in hyperbolic Pascal triangles, Analele Stiintifice Ale Universitatii Ovidius Constanta-Seria Matematica, 26(1), 189-203, 2018.

[47] Ozkan, K. (2018). Comparing Shannon entropy with Deng entropy and improved Deng entropy for measuring biodiversity when a priori data is not clear, Journal of the faculty of forestryIstanbul University, 68(2), 136-140, 2018.

[48] Pan, L.; Deng, Y. (2020). An association coefficient of belief function and its application in target recognition system, International Journal of Intelligent Systems, 35(1), 85-104, 2020.

[49] Pan, Y.; Zhang, L.; Li, Z.; Ding, L. (2019). Improved Fuzzy Bayesian Network-Based Risk Analysis With Interval-Valued Fuzzy Sets and D-S Evidence Theory, IEEE Transactions on Fuzzy Systems, DOI:10.1109/TFUZZ.2019.2929024, 2019.

[50] Qian, H.-M.; Huang, H.-Z.; Li, Y.-F. (2019). A novel single-loop procedure for time-variant reliability analysis based on Kriging model, Applied Mathematical Modelling, 75, 735-748, 2019.

[51] Rényi, A. (1961). On measures of entropy and information, Proceedings of the Fourth Berkeley Symposium on Mathematical Statistics and Probability, Volume 1: Contributions to the Theory of Statistics, The Regents of the University of California 1961.

[52] Ristic, B.; Smets, P. (2005). Target classification approach based on the belief function theory, IEEE Transactions on Aerospace and Electronic Systems, 41(2), 574-583, 2005.

[53] Robledo, A. (2013). Generalized Statistical Mechanics at the Onset of Chaos, IEEE Transactions on Aerospace and Electronic Systems, 15(12), 5178-5222, 2013.

[54] Romagnoli, S. (2019). A vague multidimensional dependency structure: Conditional versus Unconditional fuzzy copula models, IEEE Transactions on Aerospace and Electronic Systems, 512, 1202-1213, 2019.

[55] Schubert, J. (2011). Conflict management in Dempster-Shafer theory using the degree of falsity, International Journal of Approximate Reasoning, 52(3), 449-460, 2011.

[56] Seiti, H.; Hafezalkotob, A.; Najafi, S.E.; Khalaj, M. (2018). A risk-based fuzzy evidential framework for FMEA analysis under uncertainty: An interval-valued DS approach, Journal of Intelligent \& Fuzzy Systems, 35(2), 1419-1430, 2018.

[57] Shafer, G. (1967). A mathematical theory of evidence, Princeton university press, 42, 1967.

[58] Sheikholeslami, M.; Jafaryar, M.; Shafee, A.; Li, Z.; Haq, R. (2019). Heat transfer of nanoparticles employing innovative turbulator considering entropy generation, International Journal of Heat and Mass Transfer, 136, 1233-1240, 2019. 
[59] Song, Y.; Deng, Y. (2019). A new soft likelihood function based on power ordered weighted average operator, International Journal of Intelligent Systems, 34(11), 2988-2999, 2019.

[60] Song, Y.; Deng, Y. (2019). Divergence measure of belief function and its application in data fusion, IEEE ACCESS, 7, 107465-107472, 2019.

[61] Tsallis, C. (1988). Possible generalization of Boltzmann-Gibbs statistics, Journal of statistical physics, 52(1-2), 479-487, 1988.

[62] Tsallis, C.; Gellmann, M.; Sato, Y. (2005). Asymptotically scale-invariant occupancy of phase space makes the entropy Sq extensive, Proceedings of the National Academy of Sciences of the United States of America, 102(43), 15377-15382, 2005.

[63] Tuğal, I. (2019). Karcı and Shannon entropies and their effects on centrality of social networks, Physica A: Statistical Mechanics and its Applications, 523, 352-363, 2019.

[64] Velarde, C.; Robledo, A. (2015). Pascal (Yang Hui) triangles and power laws in the logistic map, Journal of Physics Conference Series, 604, 012018, 2015.

[65] Wang, H.; Fang, Y.-P.; Zio, E. (2019). Risk Assessment of an Electrical Power System Considering the Influence of Traffic Congestion on a Hypothetical Scenario of Electrified Transportation System in New York Stat, IEEE Transactions on Intelligent Transportation Systems, doi:10.1109/TITS.2019.2955359, 2019.

[66] Wang, D.; Gao, J.; Wei, D. (2019). A New Belief Entropy Based on Deng Entropy, Entropy, 21(10), doi:10.3390/e21100987, 2019.

[67] Wang, T.; Wang, J.; Ming, J.; Sun, Z.; Wei, C.; Lu, C.; Pérez-Jiménez, M.J. (2018). Application of neural-like $\mathrm{P}$ systems with state values for power coordination of photovoltaic/battery microgrids, IEEE ACCESS, 6, 46630-46642, 2018.

[68] Wang, T.; Wei, X.; Huang, T.; Wang, J.; Peng, H.; Pérez-Jiménez, M. J.; Valencia-Cabrera, L. (2019). Modeling fault propagation paths in power systems: A new framework based on event SNP systems with neurotransmitter concentration, IEEE ACCESS, 7, 12798-12808, 2019.

[69] Wang, T.; Wei, X.; Huang, T.; Wang, J.; Valencia-Cabrera, L.; Fan, Z.; Pérez-Jiménez, M. J. (2019). Cascading Failures Analysis Considering Extreme Virus Propagation of Cyber-Physical Systems in Smart Grids, Complexity, 2019, 7428458, 2019.

[70] Wei, B.; Feng, X.; Yang, S. (2019). Fully Distributed Synchronization of Dynamic Networked Systems with Adaptive Nonlinear Couplings, IEEE Transactions on Cybernetics, DOI:10.1109/TCYB.2019.2944971, 2019.

[71] Wei, B.; Feng, X.; Yang, S. (2019). Synchronization in Kuramoto Oscillator Networks With Sampled-Data Updating Law, IEEE Transactions on Cybernetics, DOI:10.1109/TCYB.2019.2940987, 2019.

[72] Wen, T.; Deng, Y. (2020). The vulnerability of communities in complex networks: An entropy approach, Reliability Engineering \& System Safety, 196, 106782, 2020.

[73] Xiao, F. (2019). EFMCDM: Evidential fuzzy multicriteria decision making based on belief entropy, IEEE Transactions on Fuzzy Systems, DOI: 10.1109/TFUZZ.2019.2936368, 2019.

[74] Xiao, F. (2019). Generalization of Dempster-Shafer theory: A complex mass function, Applied Intelligence, DOI: 10.1007/s10489-019-01617-y, 2019.

[75] Xiao, F. (2020). A new divergence measure for belief functions in D-S evidence theory for multisensor data fusion, Information Sciences, 514, 462-483, 2020. 
[76] Yager, R.R. (2014). On the maximum entropy negation of a probability distribution, IEEE Transactions on Fuzzy Systems, 23(5), 1899-1902, 2014.

[77] Yager, R. R. (2019). Generalized Dempster-Shafer Structures, IEEE Transactions on Fuzzy Systems, 27(3), 428-435, 2019.

[78] Yuan, R.; Tang, M.; Wang, H.; Li, H. (2019). A Reliability Analysis Method of Accelerated Performance Degradation Based on Bayesian Strategy, IEEE Access, 7, 169047-169054, 2019.

[79] Zhao, H.; Xie, Z. (2014). Preliminary study of cellular automat on mobile computing application, Applied Mechanics and Materials, 519, 838-841, 2014.

[80] Zhou, M.; Liu, X.; Chen, Y.; Yang, J. (2018). Evidential reasoning rule for MADM with both weights and reliabilities in group decision making, Knowledge-Based Systems, 143, 142-161, 2018.

[81] Zhou, M.; Liu, X.; Yang, J.; Chen, Y.; Wu, J. (2019). Evidential reasoning approach with multiple kinds of attributes and entropy-based weight assignment, Knowledge-Based Systems, 163, 358$375,2019$.

[82] Zurek, W. H. (2018). Complexity, entropy and the physics of information, CRC Press, 2018.

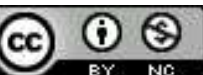

Copyright (C)2020 by the authors. Licensee Agora University, Oradea, Romania.

This is an open access article distributed under the terms and conditions of the Creative Commons Attribution-NonCommercial 4.0 International License.

Journal's webpage: http://univagora.ro/jour/index.php/ijccc/

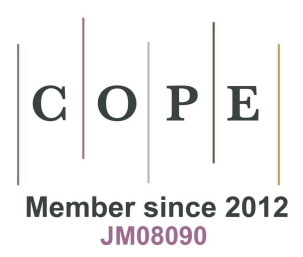

This journal is a member of, and subscribes to the principles of,

the Committee on Publication Ethics (COPE).

https://publicationethics.org/members/international-journal-computers-communications-and-control

Cite this paper as:

Gao, X.; Deng, Y.(2020). The Pseudo-Pascal Triangle of Maximum Deng Entropy, International Journal of Computers Communications \& Control, 15(1), 1006, 2020. https://doi.org/10.15837/ijccc.2020.1.3735 